\title{
Performed Study Subject Milestone Informed Consent Date
}

National Cancer Institute

\section{Source}

National Cancer Institute. Performed Study Subject Milestone Informed Consent Date. NCI Thesaurus. Code C93964.

The date (and time) the study subject gives official consent by signing the official consent form. 